\title{
Genetic Diversity of Mastitis-Associated Klebsiella pneumoniae in Dairy Cows
}

\author{
G. G. Paulin-Curlee, ${ }^{*}$ R. S. Singer, ${ }^{\star}$ S. Sreevatsan,† R. Isaacson, ${ }^{\star}$ J. Reneau,‡ D. Foster,‡ and R. Bey ${ }^{\star 1}$ \\ *Department of Veterinary and Biomedical Sciences, \\ †Department of Veterinary Population Medicine, and \\ ‡Department of Animal Science, University of Minnesota, St. Paul 55108
}

\section{ABSTRACT}

The objectives of this study were to determine the level of genetic diversity of Klebsiella pneumoniae isolated from clinical mastitis cases and to define genotypes most commonly associated with the disease. Individual quarter milk samples were collected from a single privately owned dairy herd over a 2-yr period and submitted to the Laboratory for Udder Health, Minnesota Veterinary Diagnostic Laboratory, University of Minnesota, for bacteriological culture. Eighty-four $K$. pneumoniae isolates were obtained and fingerprinted by repetitive DNA sequence PCR, 43 by pulsed-field gel electrophoresis (PFGE), and 29 by multilocus sequence typing (MLST). Significant genetic diversity was observed among the isolates regardless of the fingerprinting method used. Simpson's diversity index was 93.5, 96.1, and $97.0 \%$ when analyzed by repetitive DNA sequence PCR $(\mathrm{n}=84)$, pulse field gel electrophoresis $(\mathrm{n}=$ 43 ), and MLST ( $n=29$ ), respectively. In some cases more than 1 genotype was obtained from a single milk sample originating from an individual quarter. The majority of infections were observed during the winter and accounted for $69.0 \%$ of $K$. pneumoniae mastitis cases. There was a negative correlation between a matrix of fingerprints similarity and a matrix of temporal distances. The MLST results revealed 5 new and novel allelic types, which have not been previously reported in the MLST database. Three isolates shared MLST types with human clinical isolates, raising the possibility that some $K$. pneumoniae isolates, of bovine origin, may be capable of causing disease in humans. There were 21 genotypes present within the herd, and there was no evidence for nonrandom distribution of genotypes uniquely associated with mastitis. We have shown, using 3 distinct genotyping methods, that $K$. pneumoniae isolated from clinical mastitis within a single dairy herd is caused by a genetically diverse popula-

Received November 20, 2006.

Accepted April 18, 2007.

${ }^{1}$ Corresponding author: beyxx001@umn.edu tion and that multiple genotypes can be isolated from a mastitic quarter. The data suggest that mastitis can be caused by a variety of $K$. pneumoniae genotypes. Diverse genotypes may have different levels of invasiveness and virulence and may originate from various sources within the dairy.

Key words: mastitis, Klebsiella, diversity

\section{INTRODUCTION}

Mastitis remains one of the most important diseases among dairy herds despite the widespread use of control programs including techniques such as teat dipping, dry cow therapy, and segregation of infected animals (Esslemont and Kossaibati, 1997). As a result of control programs for contagious mastitis pathogens (Staphylococcus aureus, Streptococcus agalactiae, Mycoplasma species), mastitis caused by environmental pathogens (coliform bacteria, fecal streptococci, Streptococcus dysgalactiae and uberis) is now the primary disease in wellmanaged dairy herds with low SCC in bulk tank milk (Barkema et al., 1998).

Coliform bacteria can cause clinical or subclinical mastitis. Cows with clinical mastitis can have abnormal milk, swollen quarters, anorexia, and fever. In subclinical mastitis there are no visible signs of disease. Inflammation can only be monitored by in vitro diagnostic tests. Previously, coliform bacteria have been shown to be responsible for approximately half of the acute mastitis episodes (Erskine et al., 1991). The control and prevention of coliform mastitis remains a challenge to dairy producers. Control measures that are applied for contagious mastitis pathogens are often ineffective in controlling mastitis caused by coliform bacteria. Presently, the most efficient strategy for controlling coliform mastitis is prevention through appropriate management and prophylactic immunization (Bradley and Green, 2000).

The number of mastitis cases caused by coliform bacteria has increased worldwide (Schukken et al., 1989). Specifically, $K$. pneumoniae has been found to affect many dairy herds (Silva and Costa, 2001; Sampimon 
et al., 2006). Braman et al. (1973) found 33 capsular types of $K$. pneumoniae in 12 herds. Within each herd as many as 13 capsular types were found, indicating there were different variants of the same organism present. In another study, Kikuchi et al. (1995) reported diversity in plasmid profiles among $47 \mathrm{~K}$. pneumoniae isolates from mastitic milk. However, plasmid profile typing is not as discriminatory as chromosomal DNA genotyping because plasmids may be lost, gained spontaneously, or exchanged between strains, making it difficult to determine the potential number of variants present.

Mastitis caused by $K$. pneumoniae can be particularly severe compared with mastitis caused by Escherichia coli, due to its poor response to antibiotic therapy, rapid evolution to toxic shock, and death (Silva and Costa, 2001). Infection of the gland with Klebsiella species has been reported to result in higher losses than infections due $E$. coli (Grohn et al., 2004). Thus, infection with Klebsiella spp. profoundly affects the profitability of dairy farmers due to discarded milk, costs of antibiotic treatment, and extra labor, death, or culling of infected animals and decreased fertility (Hansen et al., 2004). Genotyping of $K$. pneumoniae isolated from clinical mastitis is expected to increase our understanding of the epidemiology of this organism and perhaps assist in the development of appropriate strategies for the prevention and control of udder infections in dairy cows. The goal of this study was to measure the genetic diversity of $K$. pneumoniae from cases of clinical mastitis within a dairy herd, using 3 genotyping methods [repetitive DNA sequence genotyping-PCR (rep-PCR), pulsed-field gel electrophoresis (PFGE), and multilocus sequence typing (MLST)] to fingerprint and genotype these isolates.

\section{MATERIALS AND METHODS}

\section{Klebsiella pneumoniae Isolates}

During a 2-yr period of time (January 2003 to December 2004) K. pneumoniae isolates $(\mathrm{n}=84)$ were obtained from cases of clinical mastitis from a privately owned 1,200 cow free-stall dairy herd in Wisconsin. Milk samples originating from infected quarters (swollen with abnormal milk) were sent to the Laboratory for Udder Health (University of Minnesota, St. Paul, MN) where they were processed using routine culture methods. All milk samples were cultured on MacConkey agar to isolate gram-negative bacteria and lactose positive colonies. If colonies had the gross morphologic appearance of $K$. pneumoniae they were restreaked on MacConkey agar. Klebsiella pneumoniae isolates included in the study were those in which it was the only organism isolated from the mastitic quarter. Following incuba- tion at $37^{\circ} \mathrm{C}$ for $18 \mathrm{~h}$, the identity of the isolates was verified by biochemical identification using the API $20 \mathrm{E}$ system (bioMérieux Vitek Inc., Hazelwood, MO). Three separate colonies from each clinical mastitis sample were restreaked and incubated as above. Each colony originating from the same milk sample was identified with the cow number followed by an alphabetic designation (A, B, or C) and was preserved in a glycerol-blood solution and frozen at $-80^{\circ} \mathrm{C}$.

\section{Repetitive DNA Sequence Genotyping}

Bacterial genomic DNA was extracted using PrepMan Ultra Reagent (Applied Biosystems, Foster City, CA). Briefly, frozen cultures were streaked onto MacConkey agar plates and incubated. Two individual colonies were suspended in $300 \mu \mathrm{L}$ of PBS and pelleted by centrifugation for $3 \mathrm{~min}$ at $7,500 \times g$. The supernatant was discarded, $200 \mu \mathrm{L}$ of PrepMan was added, and the tubes placed in a heat block for $10 \mathrm{~min}$ at $100^{\circ} \mathrm{C}$. After incubation, the solution was fractionated by centrifugation for $3 \mathrm{~min}$ at $7,500 \times \mathrm{g}$, and the supernatant containing DNA and an equal amount of sterile DNasefree water was added. The rep-PCR fingerprints were obtained using a boxA1R primer (5'CTACGGCAAGGCGACGCTGACG $\left.3^{\prime}\right)$. The $25-\mu \mathrm{L}$ PCR mixture contained $25 \mathrm{mM} \mathrm{MgCl}, 10 \times$ Buffer II, $25 \mathrm{pmol}$ of boxA1R primer, $100 \mathrm{~m} M$ dNTP mix, AmpliTaq Gold DNA polymerase, and $2 \mu \mathrm{L}$ of DNA template. Thermocycler PCR conditions were $95^{\circ} \mathrm{C}$ for $7 \mathrm{~min}$, followed by 30 cycles consisting of $94^{\circ} \mathrm{C}$ for $1 \mathrm{~min}, 66^{\circ} \mathrm{C}$ for $8 \mathrm{~min}, 71^{\circ} \mathrm{C}$ for 1 min, and an extension of $71^{\circ} \mathrm{C}$ for 15 min followed by storage at $4^{\circ} \mathrm{C}$. These conditions were previously described by Goldberg et al. (2006). Samples were separated by electrophoresis using a $2 \%$ agarose gel at $4^{\circ} \mathrm{C}$ for 5 to $6 \mathrm{~h}$ at $62 \mathrm{~V}(6 \mathrm{~V} / \mathrm{cm})$ and stained in ethidium bromide/1× Tris-acetate-EDTA buffer. Gel images were electronically captured using Labworks 4.0 Image Acquisition and Analysis Software (UVP Inc., Upland, CA).

\section{Pulsed-Field Gel Electrophoresis}

The PFGE protocol was based on procedures previously described by Cho et al. (2006) in addition to those described in the CHEF Genomic DNA Plug Kit (BioRad, Hercules, CA). Klebsiella pneumoniae isolates were grown overnight in $10 \mathrm{~mL}$ of brain heart infusion broth at $37^{\circ} \mathrm{C}$. Cells were pelleted by centrifugation and resuspended in $10 \mathrm{~mL}$ of cell suspension buffer $(100 \mathrm{mM}$ Tris:100 mM EDTA, $\mathrm{pH}$ 8.0) followed by centrifugation. The cells were washed a second time and resuspended in $5 \mathrm{~mL}$ of cell suspension buffer. Agarose plugs were prepared by adding $250 \mu \mathrm{L}$ of $2 \%$ CleanCut agarose 
(BioRad) to $250 \mu \mathrm{L}$ of the cell suspension with gentle mixing at $56^{\circ} \mathrm{C}$. One hundred microliters of this mixture was transferred to plug molds and allowed to solidify. The solidified agarose plugs were incubated in $300 \mu \mathrm{L}$ of freshly prepared lysozyme solution $(0.01 \mathrm{mg} / \mu \mathrm{L}$ final concentration) for $2 \mathrm{~h}$ at $37^{\circ} \mathrm{C}$. After this, plugs were incubated overnight at $56^{\circ} \mathrm{C}$ in proteinase $\mathrm{K}$ solution $(0.24 \mathrm{U} / \mu \mathrm{L}$ final concentration). Following incubation, the plugs were washed with sterile water with TrisEDTA buffer $\mathrm{pH} 8.0$ at $50^{\circ} \mathrm{C}$. After washing, half of the plugs were digested with $\mathrm{Xba \textrm {I }}$ (New England BioLabs Inc., Beverly, MA) for 1.5 to $2 \mathrm{~h}$ at $37^{\circ} \mathrm{C}$. After digestion, the $X b a$ buffer was removed, and about one-quarter of each plug was loaded into each well. A lambda DNA ladder (New England BioLabs) was used as a molecular size standard. Electrophoresis was carried out in $0.5 \times$ Tris-borate-EDTA buffer at $14^{\circ} \mathrm{C}$ with an initial switch time of $1 \mathrm{~s}$ and a final switch time of $30 \mathrm{~s}$ for $23 \mathrm{~h}$ at $6 \mathrm{~V} / \mathrm{cm}$. The gel was stained with ethidium bromide and destained with distilled water. Gel images were viewed and captured with Labworks 4.0 Image Acquisition and Analysis Software (UVP Inc.).

\section{Rep-PCR and PFGE Fingerprint Analysis}

Gel images were analyzed using BioNumerics software version 4.0 (Applied Maths BVBA, Sint-MartensLatem, Belgium). For rep-PCR, the band positions in each gel were normalized using the $1 \mathrm{~kb}$ DNA ladder (Hi-Lo DNA Marker, Minnesota Molecular Inc., Minneapolis, MN), and for PFGE the DNA band normalization was accomplished by using the 1,000 kb lambda DNA ladder (New England BioLabs Inc., Beverly, MA). Band matching and isolate similarity using rep-PCR and PFGE was accomplished using Dice band-based coefficient of similarity, which provides the most accurate similarity results when compared with visual inspection of the fingerprint patterns (Carriço et al., 2005).

The Dice similarity coefficient formula used in the analysis was

$$
S_{i j}=\frac{2 n_{i j}}{2 n_{i j}+n_{i}+n_{j}}
$$

where $S_{i j}=$ similarity; $n_{i}=$ number of unique bands occurring in pattern $i ; n_{j}=$ number of unique bands occurring in pattern $j$; and $n_{i j}=$ number of bands shared between the 2 patterns.

Tolerance and optimization settings were 1.5 and $1.0 \%$ for rep-PCR and 3.0 and $1.5 \%$ for PFGE. These settings were determined by evaluating different ranges ( 0 to $6 \%$ ) and selecting the ones with greatest accuracy when compared with visual inspection of fingerprints in the gel picture (Carriço et al., 2005). A dendrogram was constructed using the unweighted pair group method with arithmetic means, which employs a sequential clustering algorithm in which the relationships are identified in order of similarity and the dendrogram is built in a stepwise manner (Durbin et al., 1998). The PCR reproducibility was verified from 16 distinct isolates by freshly extracting DNA 4 separate times. A dendrogram containing 4 fingerprints of the same isolate was constructed for $K$. pneumoniae using the BioNumerics software and the settings mentioned above. The percent similarity among fingerprints for each dendrogram was recorded and the average calculated across dendrograms. Reproducibility showed an average of $93.6 \%$ fingerprint similarity for the same isolate and was used, along with cluster pattern, to conservatively establish the $90 \%$ similarity cut-off for genotype definition used in this study. No other mathematical analysis was used in establishing the $90 \%$ cut-off.

\section{Multilocus Sequence Typing}

Amplification of 7 housekeeping genes, rpoB (betasubunit of RNA polymerase), gapA (glyceraldehyde 3phosphate dehydrogenase), $m d h$ (malate dehydrogenase), pgi (phosphoglucose isomerase), phoE (phosphorine E), infB (translation initiation factor 2), and tonB (periplasmic energy transducer), was accomplished using PCR. Two $K$. pneumoniae reference strains, MGH 78578 (ATCC 700721 and in MLST database) and strain IA565 (which is avirulent by intratracheal inoculation in mice; kindly provided by Steve Clegg, Iowa City, IA), were included as positive controls. Primer sets for gene amplification and sequencing were described previously (Diancourt et al., 2005). The PCR amplification was performed using AmpliTaq Gold DNA polymerase (Applied Biosystems) and standard thermocycler parameters. The annealing temperatures of each primer were described at the $K$. pneumoniae MLST database (http://pubmlst.org/kpneumoniae). Amplified products were purified by ultrafiltration with Montage PCR centrifugal filter devices (Millipore, Bedford, MA). Nucleotide sequencing was performed at the Advanced Genetic Analysis Center (AGAC) University of Minnesota, St. Paul. A minimum of 2 chromatograms was performed for each amplified gene. The software MEGA 3.1 (Kumar et al., 2004) was used for editing the nucleotide sequences and to evaluate the significance of branching within trees by bootstrap analysis (500 replicates) using the maximum parsimony method. Each distinct sequence within a locus was assigned a unique allele number. Each nucleotide sequence was analyzed by BLASTn algorithm against the $K$. pneumoniae MLST database (http://pubmlst.org/kpneumon- 
iae) in search of a matching allele number. Nucleotide sequences having no matching allele in the MLST database were considered new and had their respective allele numbers and sequence type (ST) assigned by the curator of the $K$. pneumoniae MLST database Web site. Once all of the allele numbers and sequence types were assigned, cluster analysis was performed by BioNumerics software version 4.0 (Applied Maths BVBA, Martens-Latem, Belgium) using categorical coefficient. In this method, alleles containing identical nucleotide sequence were considered a match and alleles containing any nucleotide difference (irrespective of the number of repeats) were considered a no match. Additionally, the numbers assigned to each allele type does not imply any relationship between ST.

\section{Statistical Analysis}

Spearman rank coefficient was used to calculate the correlation between a matrix of fingerprints similarity and a matrix of temporal distances (number of days between dates of sample collection) using the MacAnova statistical analysis program (School of Statistics, University of Minnesota, Minneapolis, MN). A $P$-value of $\leq 0.05$ was considered significant for the temporal correlation. Simpson's diversity index was used for measurement of genetic diversity and discriminatory power of the typing techniques. Calculation of Simpson's diversity index was performed using the BioNumerics software and it was based on the following formula:

$$
\mathrm{D}=1-\frac{1}{N(N-1)} \sum_{j=1}^{S} n_{j}\left(n_{j}-1\right)
$$

where $N$ is the total number of isolates in the sample population, $S$ the total number of genotypes described, and $n_{j}$ is the number of isolates belonging to the $j$ th genotype. The genetic diversity is based on the probability that $2 \mathrm{~K}$. pneumoniae isolates chosen at random will be of a different genotype. Higher Simpson's diversity index indicates a high discriminatory power of the technique and genetic diversity within the population (Hunter and Gaston, 1988).

\section{RESULTS AND DISCUSSION}

\section{Repetitive DNA Sequence Genotyping}

A total of $84 \mathrm{~K}$. pneumoniae mastitis isolates, 3 isolates per clinical case, from a single dairy farm were fingerprinted by rep-PCR. A positive (K. pneumoniae) control and a negative control (no DNA) were included in each PCR reaction. The number of $K$. pneumoniae- associated mastitis cases was higher during the winter months, accounting for $69.0 \%$ of the cases. The remaining $K$. pneumoniae mastitis cases occurred during summer (17.2\%) and fall (13.8\%). Reports on the occurrence of Klebsiella species mastitis at a determined season of the year are lacking. However, the percentage of mastitis caused by gram-negative bacteria has been reported to be higher during summer and fall (Hogan et al., 1989). Additionally, a higher incidence of SCC, an indicator of mastitis, has been found during summer (Norman et al., 2000). Factors such as confinement in closed installations, stage of lactation, and changes in dairy management (increased number of cows present, bedding material, and frequency of bedding change, alley scrapping, overcrowding, and the cow's preparation before and after milking) could have influenced the higher percentage of $K$. pneumoniae mastitis in the winter months.

Using a 90\% cut-off for similarity in the rep-PCR banding patterns, 21 distinct fingerprint patterns were identified among the 84 isolates and were assigned a genotype. The Simpson's diversity index was 93.5\%, indicating a wide genetic diversity and a high degree of discrimination capability of rep-PCR. Each genotype was composed of between 1 and 12 isolates. Analysis of 3 distinct isolates originating from the same cow showed identical rep-PCR patterns $46.4 \%$ of the time. Two of the colonies had the same genotype pattern $35.7 \%$ of the time. The remaining $17.9 \%$ showed different genotype patterns for all isolates indicating that more than one $K$. pneumoniae variant can be isolated from the mastitic bovine mammary gland. The possibility of sample contamination exists, but to reduce this possibility only those samples from which $K$. pneumoniae was the only organism isolated were included in this study and the individual responsible for sample collection had been extensively trained in how to aseptically collect milk samples. It is possible that errors inherent in the PCR technique may have partially accounted for the different genotypes encountered in $K$. pneumoniae isolated from the same mastitis case sample.

Two dairy cows participating in this study died with acute mastitis. Isolates originating from cows that died (716 and 1481) possessed rep-PCR genotypes distinct from each other. Other cows infected with the same genotypes did not die and remained in the herd. It is possible that in addition to Klebsiella's virulence traits, factors such as stage of lactation, immune and nutritional status, age, and genetics may have contributed to the severity of these mastitis cases.

Spearman rank coefficient was used to calculate the correlation between a matrix of genetic similarity and a matrix of temporal distances. The association of the 
genetic similarity matrix and the temporal distance matrix could provide more information on the dynamics of $K$. pneumoniae mastitis. The results showed a significant negative association between the 2 matrices $(\mathrm{R}=-0.1525 ; P=0.0246)$, indicating that isolates that were collected closer in time had higher similarity than those collected farther apart in time. The correlation was not very strong but was significantly different from zero. The great genetic diversity may explain the low correlation observed. The results suggest that temporal distance seems to play a role in the $K$. pneumoniae genotypes isolated from clinical cases of mastitis.

\section{Pulsed-Field Gel Electrophoresis Genotyping}

To verify results obtained by rep-PCR, a subset of 43 isolates was selected to be typed by PFGE. The enzyme $X b a \mathrm{I}$ was used for PFGE typing because it is recommended by the Centers for Disease Control and Prevention. The selection of isolates for PFGE typing was based on similarities in rep-PCR pattern. Out of 21 repPCR genotypes, 15 were represented in PFGE. We also included isolates that originated from the same clinical case: 3 per case $(n=12), 2$ per case $(n=16)$. The remaining isolates were singly represented $(\mathrm{n}=15)$, meaning that only 1 isolate per clinical case was analyzed. Using a 90\% cut-off, 23 distinct PFGE genotypes were detected in the 43 isolates and each genotype contained 1 to 6 isolates. Klebsiella pneumoniae isolates typed by PFGE showed greater genetic diversity than observed with rep-PCR. It was interesting to note that, with both techniques, some of the isolates from the same clinical mastitis cases exhibited more than 1 genotype pattern ( 29.0 and $17.9 \%$ by PFGE and rep-PCR, respectively), supporting the result that more than one $K$. pneumoniae variant can be isolated from the infected mammary gland and confirming the high discriminatory power of PFGE. Isolates that originated from the same mastitis cases $(45.2 \%)$ clustered similarly by repPCR and by PFGE. The Simpson's diversity index for PFGE was $96.1 \%$, indicating the wide diversity of $K$. pneumoniae within the dairy herd. Once again, isolates from the 2 cows that died of acute mastitis showed distinct genotype patterns by PFGE. The isolates clustering with the acute mastitis cases by PFGE were different from those identified by rep-PCR. Pulsed-field gel electrophoresis showed higher discriminatory power (Simpson's diversity index $=96.1 \%$ ) when compared with rep-PCR (Simpson's diversity index $=93.5 \%$ ).

\section{Multilocus Sequence Typing Genotyping}

Due to the great genetic diversity observed and the possible subjective interpretation of the results by DNA band-based techniques, genetic diversity among the $K$. pneumoniae isolates was analyzed using MLST, which is characterized as having high reproducibility and discriminatory power. A subset of 29 isolates was analyzed by MLST. Thirteen PFGE genotypes were typed by MLST, which included isolates from the same mastitis cases, 3 per case $(n=9)$ and 2 per case $(n=8)$, and single isolates from different mastitis cases $(\mathrm{n}=12)$. Twenty-nine $K$. pneumoniae mastitis isolates and 2 type strains used as positive controls (MGH78578 and IA565) were analyzed by MLST. This analysis resulted in a total of 23 ST measured as a composite of 7 genetic loci studied. Excluding ST formed by the 2 reference strains, MLST analysis had a Simpson's diversity index of $97.0 \%$. This high level of diversity is supported by bootstrap analysis, which also had high values. Clinical mastitis isolates composed $21 \mathrm{ST}$, whereas 2 distinct ST composed the 2 reference strains. As in rep-PCR and PFGE, isolates originating from the same clinical mastitis case had distinct ST. Five cases of mastitis with 2 isolates per case exhibited distinct ST for each isolate. Three isolates originating from the same clinical mastitis sample presented the same ST. Comparable variability was shown by rep-PCR for the same set of samples in which 3 of them had the same rep-PCR genotype and 2 had distinct rep-PCR genotype. Two samples having distinct ST by MLST showed the same genotype by rep-PCR. One of the isolates (716A), which caused acute mastitis (evolution to toxemic shock and death), shared the same ST with isolate 2261A. Both isolates clustered together by MLST and rep-PCR.

Nucleotide variation was observed in all 7 genes with each gene having 4 to 13 distinct allelic types. Five ST $(98,104,110,112$, and 116) and 5 of the allelic types found were new, novel, and did not present patterns found in the MLST database for K. pneumoniae (http:// pubmlst.org/kpneumoniae). The genes rpoB, gapA, and infB contained the least amount of allelic variation with each having 4 to 5 different allelic types. The $m d h$ and pgi genes contained 6 allelic types. Genes $p h o E$ and ton $B$ contained the largest amount of allele variation with 9 and 13 allelic types, respectively. The novel ST and allelic types are shown in Table 1. Novel allelic types were found for all genes except rpoB, pgi, and $p h o E$. Two new allelic types were found for ton $B$ and gapA; $m d h$ and infB had one new allelic type each. Considering that all isolates originated from a single herd, a significant amount of nucleotide diversity was observed in the housekeeping genes. Our results support those previously reported by Diancourt et al. (2005) that ton $B$ exhibited the greatest amount of nucleotide variation. Three of the clinical mastitis isolates from cows 716, 2,261, and 3,825 shared ST (14 and 45) with human clinical isolates and one fecal isolate listed in 
Table 1. Novel sequence types (ST) and allelic profiles

\begin{tabular}{lcccccccc}
\hline ST & rpoB & gapA & $m d h$ & pgi & phoE & infB & tonB & Isolate \\
\hline 98 & 4 & 1 & $20^{2}$ & 1 & 1 & 6 & 1 & $5250 \mathrm{~A}$ \\
104 & 1 & 2 & 1 & 1 & 2 & 3 & $43^{2}$ & $2794 \mathrm{~A}$ \\
110 & 1 & 2 & 1 & 3 & 8 & 6 & $44^{2}$ & $6010 \mathrm{~A}$ \\
112 & 1 & 2 & 6 & 1 & 9 & $17^{2}$ & 4 & $794 \mathrm{C}$ \\
116 & 1 & $15^{2}$ & 1 & 18 & 1 & 1 & 4 & $1994 \mathrm{~A}$ \\
\hline
\end{tabular}

${ }^{1}$ rpoB = beta-subunit of RNA polymerase; gapA = glyceraldehyde 3-phosphate dehydrogenase; $m d h=$ malate dehydrogenase, $p g i=$ phosphoglucose isomerase, $p h o E=$ phosphorine $\mathrm{E}$, inf $B=$ translation initiation factor $2, \operatorname{ton} B=$ periplasmic energy transducer; http://pubmlst.org/lpneumoniae/.

${ }^{2}$ Novel allelic type.

the MLST database for $K$. pneumoniae. It is possible that isolates from clinical cases of mastitis have virulence attributes capable of causing human disease.

\section{Association of Rep-PCR with MLST and PFGE}

When comparing the 31 isolates (29 bovine and 2 human) analyzed by MLST, PFGE, and rep-PCR, partial concordance among the techniques was found. The 23 ST determined by MLST were distributed in 13 genotype patterns by rep-PCR and 18 genotype patterns by PFGE. Dendrograms using the same samples for analysis by rep-PCR and PFGE were constructed using unweighted pair group method with arithmetic mean, and Figure 1 depicts the level of similarity (100 to 70\%) of isolates driven by rep-PCR fingerprints. The PFGE and rep-PCR genotype patterns composed a variety of ST, and a specific ST did not correlate to a specific genotype fingerprint. Due to the extensive nucleotide variation (between 4 and 13 distinct nucleotide polymorphisms), only a few ST composed more than 1 isolate; thus, it was difficult to associate a specific ST with a specific genotype pattern.

Despite the fact that the 3 genotyping techniques showed a great genetic diversity, the different isolates did not necessarily cluster in the same order. Five sets of isolates clustered by rep-PCR and by MLST. Four isolates with distinct ST also showed a distinct genotype by rep-PCR. Three isolate sets clustered similarly by rep-PCR and PFGE, and 4 isolates (MGH 78578, IA $565,1994 \mathrm{~A}$, and 5250B) had unique genotypes by both techniques. Each technique measures different attributes; they all possess specific reproducibility issues and discriminatory powers. Thus, it is possible and probable that not all isolates would be clustered similarly. The rep-PCR method is based on the amplification of repetitive elements in the bacterial chromosome, whereas RFLP observed in the entire chromosome is the basis for PFGE (Martin et al., 1992; Gori et al., 1996). The MLST method depends on nucleotide polymorphisms and the allelic profiles of 7 housekeeping genes (Diancourt et al., 2005). Thus, it seems unreason- able to draw mathematical correlations among these 3 distinct methods. It is possible that selecting subsets of isolates based on rep-PCR fingerprints to be analyzed by PFGE and MLST may have been biased and contributed to the low concordance among techniques. Higher correlation among techniques may have been found had we typed all $84 \mathrm{~K}$. pneumoniae isolates by PFGE and MLST. Two different typing methods were used to verify genetic variation in $K$. pneumoniae clinical mastitis isolates identified by rep-PCR. The MLST and PFGE techniques were the most discriminatory typing techniques in this study when compared with rep-PCR. The objective interpretation of nucleotide sequences in MLST was definitely an advantage. However, rep-PCR is fast, of low cost, easy to perform, and advantageous when analyzing a large number of isolates.

We were unable to identify 1 or 2 unique clones within a $K$. pneumoniae population associated with clinical mastitis. We have shown, with the use of 3 distinct methods (rep-PCR, PFGE, and MLST) that clinical mastitis can be caused by a genetically diverse group of $K$. pneumoniae and that more than 1 genotype can be isolated from cow(s) with clinical mastitis.

Struve and Krogfelt (2004), using a mouse infection model, found that $K$. pneumoniae isolates of environmental origin were as virulent as those isolated from a human infection. Klebsiella pneumoniae had the highest growth in bedding materials when compared with other environmental bacterial species (Zehner et al., 1986). Studies have shown that Klebsiella species had extended survival rates in the environment and were more resistant to solar radiation than Escherichia coli (McCambridge and McMeekin, 1981; Lopez-Torres et al., 1987). It is possible that the hearty and ubiquitous nature of Klebsiella associated with the particular conditions within a dairy environment contributed to the large genetic diversity observed, which is consistent with previous reports (Braman et al., 1973; Kikuchi et al., 1995). Other environmental mastitis causing bacteria, such as Streptococcus uberis, have also shown a wide genetic variation (Wieliczko et al., 2002). Similar genetic diversity has been found in isolates of $E$. coli 


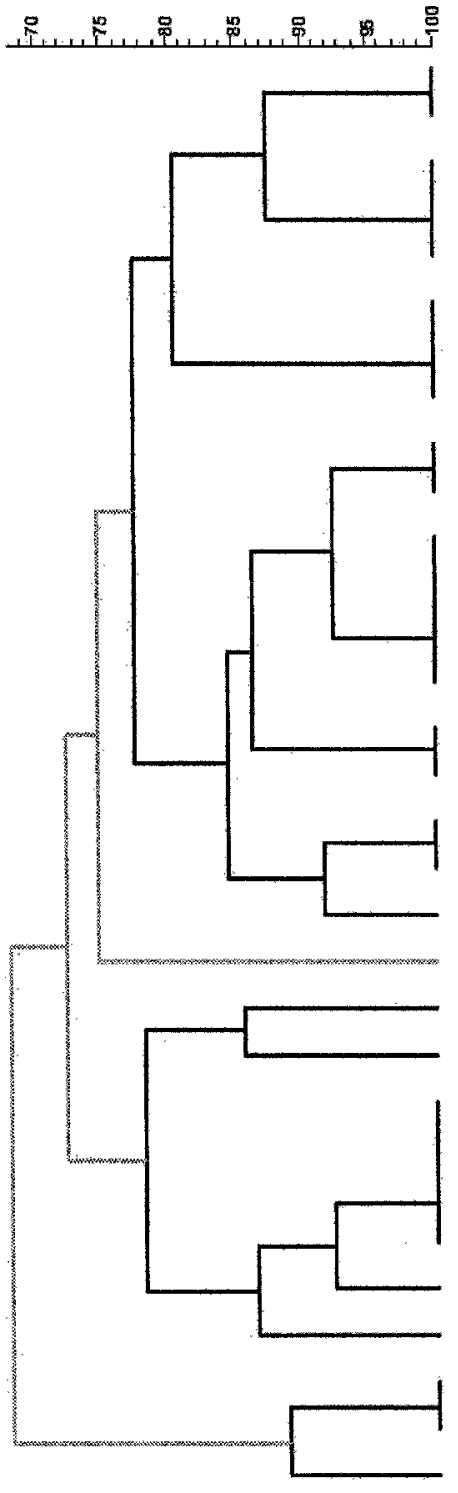

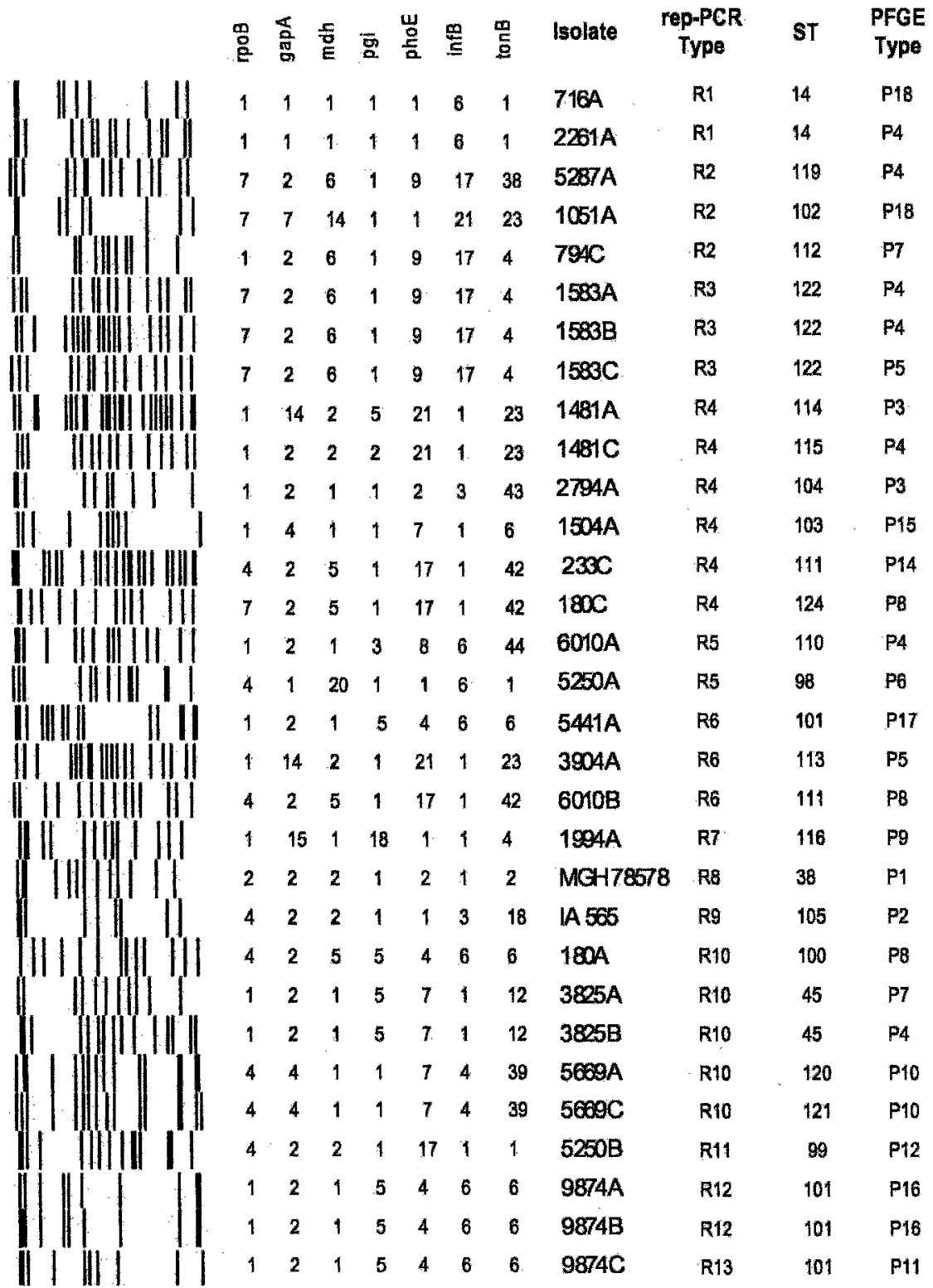

Figure 1. Repetitive DNA sequence genotyping (rep-PCR) clustering of 31 Klebsiella pneumoniae isolates. The rep-PCR pattern using boxA1R primer (BOX - group of highly conserved DNA sequences located within intergenic regions of the chromosome), pulse field gel electrophoresis (PFGE) pattern, allelic profiles, isolate identification, rep-PCR type, multilocus sequence type (ST), and PFGE type of 29 $K$. pneumoniae clinical mastitis isolates and 2 reference strains. The dendrogram was constructed on the basis of rep-PCR genotype patterns. Isolates identified by the same number but with different alphabetic designation (A, B, C) indicate separate colonies originating from the same clinical mastitis sample. Genotypes were arbitrarily assigned by technique with a capital letter and numbers for rep-PCR type (R1R13) and PFGE type (P1-P18). Multilocus sequence typing (MLST) type was designated by the ST. The horizontal scale on the left side (100 to 70 ) indicates the level of similarity in percent among fingerprints.

(Yang et al., 2004). The results of the present investigation help explain, in part, the difficulty in controlling and preventing environmental mastitis. The presence of such a genetically complex and diverse microbial population makes it difficult to target specific subpopu- lations of bacteria for control within the dairy environment. Klebsiella pneumoniae isolated from the dairy herd in this study is genetically diverse and may represent a snapshot of the diversity in the population. Still, it is possible that there are many potential sources 
of $K$. pneumoniae within a dairy herd. A larger study including isolates from several herds is necessary to confirm such findings.

Cleanliness in a dairy facility can be difficult to maintain due to the constant production of organic matter such as feces, milk, and urine and the influence of external factors such as the weather. The presence of such a genetically complex microbial population associated with the challenges of maintaining good sanitation makes it difficult to control environmental mastitis pathogens. Klebsiella pneumoniae in the environment is subject to diverse conditions (temperature, $\mathrm{pH}$, nutrients, moisture, and exposure to other microbes), which may lead to the horizontal transfer of DNA, mutations, or both, which could account for some of the genetic variation observed (Rocha, 2004). Additionally, K. pneumoniae could have variable genome sizes that account for some of the differences observed in rep-PCR and PFGE. This hypothesis is based on $E$. coli genome size variations (Bergthorsson and Ochman, 1998). The combination of all factors mentioned above may be responsible for the wide genetic variation encountered in this $K$. pneumoniae population.

The majority of molecular epidemiologic studies of $K$. pneumoniae have been performed with isolates from humans, especially outbreaks in neonatal and adult intensive care units. Contrary to our findings, $K$. pneumoniae isolated from hospitals follow a clonal pattern, and a single clone was usually associated with an outbreak. Some epidemiologic studies have reported up to 4 distinct $K$. pneumoniae clones circulating in hospital outbreaks (Dashti et al., 2006). Unlike a hospital, a dairy facility is subjected to factors that are difficult to control. Some of these factors include the weather, animal crowding, bedding management, moisture, cleanliness of installations, lack of consistency in premilking cow preparation, introduction of new animals into the herd, birds, and rodents. It is possible that a very diverse population of $K$. pneumoniae would have access to dairy facilities resulting in increased genetic diversity.

\section{CONCLUSION}

We observed great genetic diversity among the $K$. pneumoniae isolates evaluated in this study. The 3 typing techniques used in our investigation (rep-PCR, PFGE, and MLST) demonstrated that the K. pneumoniae population associated with clinical mastitis within a dairy herd is highly variable. The rep-PCR method had a slightly lower discriminatory index when compared with PFGE and MLST; however, it remains a reasonable and accurate molecular typing tool because it is relatively easy to perform especially when applied to a large number of isolates. The PFGE and MLST methods confirmed the genetic diversity results obtained by rep-PCR. The MLST results revealed 23 distinct ST and nucleotide polymorphisms. Five of the ST and 5 allelic types were new, novel, and have not been previously reported. The diversity revealed by MLST analysis and the discovery of new allelic sequences confirms the findings of rep-PCR and PFGE, which showed that there are several genotypes of $K$. pneumoniae associated with clinical mastitis within this dairy herd. The ubiquitous and opportunistic nature of $K$. pneumoniae supports the results that a genetically diverse population is capable of invading the mammary gland of dairy cows. The basis of genetic variation among $K$. pneumoniae isolates from this dairy herd has not been elucidated and needs further investigation. Because the present study included isolates from a single dairy and from a single source within the dairy (clinical mastitis cases), it may reveal only a fraction of the diversity present. Continuous monitoring of the genetic diversity of $K$. pneumoniae is of importance because it may disclose differences in virulence, epidemiology, evolution, and genetic traits that influence control and treatment. Thus, a better understanding of microbial populations within and among dairy herds may bring new information to the current management, treatment, and diagnostic procedures of mastitis.

\section{ACKNOWLEDGMENTS}

The authors wish to express their extreme appreciation to Jill Brandel, Roberta Kopel, My Yang, and Amanda Zandlo. We thank the Minnesota Veterinary Diagnostic Laboratory for their support of this project and Christopher Bingham for his help with the statistical analysis.

\section{REFERENCES}

Barkema, H. W., Y. H. Schukken, T. J. Lam, M. L. Beiboer, G. Benedictus, and A. Brand. 1998. Management practices associated with low, medium, and high somatic cell counts in bulk milk. J. Dairy Sci. 81:1917-1927.

Bergthorsson, U., and H. Ochman. 1998. Distribution of chromosome length variation in natural isolates of Escherichia coli. Mol. Biol. Evol. 15:6-16.

Bradley, A. J., and M. J. Green. 2000. A study of the incidence and significance of intramammary enterobacterial infections acquired during the dry period. J. Dairy Sci. 83:1957-1965.

Braman, S. K., R. J. Eberhart, M. A. Asbury, and G. J. Hermann. 1973. Capsular types of Klebsiella pneumoniae associated with bovine mastitis. J. Am. Vet. Med. Assoc. 162:109-111.

Carriço, J. A., F. R. Pinto, C. Simas, S. Nunes, N. G. Sousa, N. Frazao, H. de Lencastre, and J. S. Almeida. 2005. Assessment of bandbased similarity coefficients for automatic type and subtype classification of microbial isolates analyzed by pulsed-field gel electrophoresis. J. Clin. Microbiol. 43:5483-5490.

Cho, S., J. B. Bender, F. Diez-Gonzalez, C. P. Fossler, C. W. Hedberg, J. B. Kaneene, P. L. Ruegg, L. D. Warnick, and S. J. Wells. 2006. 
Prevalence and characterization of Escherichia coli $\mathrm{O} 157$ isolates from Minnesota dairy farms and county fairs. J. Food Prot. 69:252-259.

Dashti, A. A., R. Paton, and S. G. Amyes. 2006. Linkage of ciprofloxacin resistance with a single genotypic cluster of Klebsiella pneumoniae. Int. J. Antimicrob. Agents 27:73-76.

Diancourt, L., V. Passet, J. Verhoef, P. A. Grimont, and S. Brisse. 2005. Multilocus sequence typing of Klebsiella pneumoniae nosocomial isolates. J. Clin. Microbiol. 43:4178-4182.

Erskine, R. J., J. W. Tyler, M. G. Riddell Jr., and R. C. Wilson. 1991. Theory, use, and realities of efficacy and food safety of antimicrobial treatment of acute coliform mastitis. J. Am. Vet. Med. Assoc. 198:980-984.

Esslemont, R. J., and M. A. Kossaibati. 1997. Culling in 50 dairy herds in England. Vet. Rec. 140:36-39.

Goldberg, T. L., T. R. Gillespie, and R. S. Singer. 2006. Optimization of analytical parameters for inferring relationships among Escherichia coli isolates from repetitive-element PCR by maximizing correspondence with multilocus sequence typing data. Appl. Environ. Microbiol. 72:6049-6052.

Gori, A., F. Espinasse, A. Deplano, C. Nonhoff, M. H. Nicolas, and M. J. Struelens. 1996. Comparison of pulsed-field gel electrophoresis and randomly amplified DNA polymorphism analysis for typing extended-spectrum-beta-lactamase-producing Klebsiella pneumoniae. J. Clin. Microbiol. 34:2448-2453.

Grohn, Y. T., D. J. Wilson, R. N. Gonzalez, J. A. Hertl, H. Schulte, G. Bennett, and Y. H. Schukken. 2004. Effect of pathogen-specific clinical mastitis on milk yield in dairy cows. J. Dairy Sci. 87:3358-3374.

Hansen, P. J., P. Soto, and R. P. Natzke. 2004. Mastitis and fertility in cattle-Possible involvement of inflammation or immune activation in embryonic mortality. Am. J. Reprod. Immunol. $51: 294-301$.

Hogan, J. S., K. L. Smith, K. H. Hoblet, P. S. Schoenberger, D. A. Todhunter, W. D. Hueston, D. E. Pritchard, G. L. Bowman, L. E. Heider, and B. L. Brockett. 1989. Field survey of clinical mastitis in low somatic cell count herds. J. Dairy Sci. 72:1547-1556.

Hunter, P. R., and M. A. Gaston. 1988. Numerical index of the discriminatory ability of typing systems: An application of Simpson's index of diversity. J. Clin. Microbiol. 26:2465-2466.

Kikuchi, N., C. Kagota, T. Nomura, T. Hiramune, T. Takahashi, and R. Yanagawa. 1995. Plasmid profiles of Klebsiella pneumoniae isolated from bovine mastitis. Vet. Microbiol. 47:9-15.
Kumar, S., K. Tamura, and M. Nei. 2004. MEGA3: Integrated software for molecular evolutionary genetics analysis and sequence alignment. Brief Bioinform. 5:150-163.

Lopez-Torres, A. J., T. C. Hazen, and G. A. Toranzos. 1987. Distribution and in situ survival and activity of Klebsiella pneumoniae and Escherichia coli in a tropical rain forest watershed. Curr. Microbiol. 15:213-218.

Martin, B., O. Humbert, M. Camara, E. Guenzi, J. Walker, T. Mitchell, P. Andrew, M. Prudhomme, G. Alloing, and R. Hakenbeck. 1992. A highly conserved repeated DNA element located in the chromosome of Streptococcus pneumoniae. Nucleic Acids Res. 20:3479-3483.

McCambridge, J., and T. A. McMeekin. 1981. Effect of solar radiation and predacious microorganisms on survival of fecal and other bacteria. Appl. Environ. Microbiol. 41:1083-1087.

Norman, H. D., R. H. Miller, J. R. Wright, and G. R. Wiggans. 2000. Herd and state means for somatic cell count from dairy herd improvement. J. Dairy Sci. 83:2782-2788.

Rocha, E. P. 2004. Order and disorder in bacterial genomes. Curr. Opin. Microbiol. 7:519-527.

Sampimon, O. C., J. Sol, and P. A. Kock. 2006. An outbreak of Klebsiella pneumoniae mastitis. Tijdschr. Diergeneeskd. 131:2-4.

Schukken, Y. H., F. J. Grommers, D. van de Geer, and A. Brand 1989. Incidence of clinical mastitis on farms with low somatic cell counts in bulk milk. Vet. Rec. 125:60-63.

Silva, N., and G. M. Costa. 2001. An outbreak of acute bovine mastitis caused by Klebsiella pneumoniae in a dairy herd. Arq. Bras. Med. Vet. Zootec. 53:401-405.

Struve, C., and K. A. Krogfelt. 2004. Pathogenic potential of environmental Klebsiella pneumoniae isolates. Environ. Microbiol. 6:584-590.

Wieliczko, R. J., J. H. Williamson, R. T. Cursons, S. J. Lacy-Hulbert, and M. W. Woolford. 2002. Molecular typing of Streptococcus uberis strains isolated from cases of bovine mastitis. J. Dairy Sci. 85:2149-2154.

Yang, H. H., R. T. Vinopal, D. Grasso, and B. F. Smets. 2004. High diversity among environmental Escherichia coli isolates from a bovine feedlot. Appl. Environ. Microbiol. 70:1528-1536.

Zehner, M. M., R. J. Farnsworth, R. D. Appleman, K. Larntz, and J. A. Springer. 1986. Growth of environmental mastitis pathogens in various bedding materials. J. Dairy Sci. 69:1932-1941. 